OPEN ACCESS

Edited by:

Baojun Zhang,

Xi'an Jiaotong University, China

Reviewed by:

Yong Liu,

Xuzhou Medical University, China

Hou Yuzhu,

University of Chicago, United States

*Correspondence:

Dan-Li Xie

xiedl001@wmu.edu.cn

Yong-Liang Lou

lyl@wmu.edu.cn

Yi Zheng

zhengyi@wmu.edu.cn

tThese authors have contributed equally to this work

Specialty section:

This article was submitted to Cell Death and Survival,

a section of the journal

Frontiers in Cell and Developmental

Biology

Received: 27 July 2020

Accepted: 07 September 2020

Published: 08 October 2020

Citation:

Huang $X-H$, Ma $Y$, Zheng $M-M$, Chen N, Hu M-N, Wu L-Y, Zheng Y, Lou Y-L and Xie D-L (2020) NLRP3 and mTOR Reciprocally Regulate

Macrophage Phagolysosome Formation and Acidification Against

Vibrio vulnificus Infection.

Front. Cell Dev. Biol. 8:587961. doi: $10.3389 /$ fcell.2020.587961

\section{NLRP3 and mTOR Reciprocally Regulate Macrophage Phagolysosome Formation and Acidification Against Vibrio vulnificus Infection}

\author{
Xian-Hui Huang ${ }^{1,2,3+}$, Yao Ma ${ }^{1,2+}$, Meng-Meng Zheng ${ }^{1,2+}$, $\mathrm{Na}$ Chen ${ }^{1,2}, \mathrm{Mei}-\mathrm{Na} \mathrm{Hu}^{1,2}$, \\ Liu-Ying Wu 1,2, Yi Zheng $^{1,2,3 *}$, Yong-Liang Lou ${ }^{1,2,3 *}$ and Dan-Li Xie ${ }^{1,2,3 *}$ \\ ${ }^{1}$ Department of Microbiology and Immunology, School of Laboratory Medicine and Life Science, Wenzhou Medical \\ University, Wenzhou, China, ${ }^{2}$ Key Laboratory of Laboratory Medicine, Ministry of Education of China, Wenzhou, China, \\ ${ }^{3}$ Wenzhou Key Laboratory of Sanitary Microbiology, Wenzhou, China
}

The marine bacterium Vibrio vulnificus causes potentially fatal bloodstream infections, typically in patients with chronic liver diseases. The inflammatory response and antibacterial function of phagocytes are crucial for limiting bacterial infection in the human hosts. How V. vulnificus affects macrophages after phagocytosis is unclear. In this report, we found that the bactericidal activity of macrophages to internalize V. vulnificus was dependent on mammalian target of rapamycin (mTOR) and NODlike receptor (NLR) family pyrin domain containing 3 (NLRP3) interaction. Additionally, the NLRP3 expression was dependent on mTORC1 activation. Inhibited mTORC1 or absence of NLRP3 in macrophages impaired $V$. vulnificus-induced phagosome acidification and phagolysosome formation, leading to a reduction of intracellular bacterial clearance. mTORC1 signaling overactivation could increase NLRP3 expression and restore insufficient phagosome acidification. Together, these findings indicate that the intracellular bactericidal activity of macrophages responding to $V$. vulnificus infection is tightly controlled by the crosstalk of NLRP3 and mTOR and provide critical insight into the host bactericidal activity basis of clearance of $V$. vulnificus through lyso/phagosome.

Keywords: Vibrio vulnificus, NLRP3, mTOR, phagolysosome, acidification, bactericidal activity

\section{INTRODUCTION}

Vibrio vulnificus is a Gram-negative bacterium that causes primary septicemia, inflammationmediated septic shock, and necrotizing wound infection (Blake et al., 1979; Park and Lee, 2018; Baker-Austin and Oliver, 2020). Cases of V. vulnificus infection have been reported worldwide, including the United States, China, Australia, Germany, Korea, and Japan (Zhao et al., 2015; Heng et al., 2017). Infection with this bacterium is related to V. vulnificus contaminated raw seafood consumption or an open wound that was exposed to warm seawater containing this bacteria (BakerAustin and Oliver, 2020). V. vulnificus infection can lead to death by developing an overwhelming primary sepsis (Chou et al., 2010; Baker-Austin and Oliver, 2018, 2020). The pathogenesis of $V$. vulnificus infection, including the virulence factors, and cell death and inflammation induced 
by this bacteria have been well-defined (Gulig et al., 2005; Jeong and Satchell, 2012; Arezes et al., 2015; Baker-Austin and Oliver, 2018). However, little is known about the underlying mechanisms by which molecules in human hosts regulate the host's innate immunity-mediated anti- $V$. vulnificus defense.

Phagocytes, such as neutrophils and macrophages, are the frontline cells of host defense against pathogenic microbial invasion (Russell et al., 2009; Diacovich and Gorvel, 2010). Macrophages play an essential role in the uptake and clearance of pathogens and detect harmful stimuli, including various bacterial components and infectious agents that appear during bacterial infection, via the receptors or phagocytosis of microbes (Ishii et al., 2008). Subsequently, macrophages are activated and produce various inflammatory cytokines, chemokines, and antimicrobial peptides. These macrophage-produced signaling molecules facilitate the activation of innate immune signaling pathways, such as transcriptional regulation signaling, inflammasome activation signaling, and mammalian target of rapamycin (mTOR) signaling (Benoit et al., 2008; Bauernfeind et al., 2009; Weichhart et al., 2015). mTOR is a serine/threonine kinase that regulates various effector responses of both innate and adaptive immune cells. mTOR forms two unique complexes: mTORC1 and mTORC2. Both complexes act as crucial regulators of multiple cellular processes in the innate immune cells, including protein translation, cytokine production, metabolism, cell survival, macrophage polarization, and self-renewal (Pan et al., 2012; Byles et al., 2013; Weichhart et al., 2015; Deng et al., 2017). Many extracellular signals can induce mTORC1 and mTORC2 activation in innate immune cells, including toll-like receptors (TLR), cytokines, and growth factors. mTOR signaling also plays a dynamic role in the regulation of macrophage activation, which further drives macrophage polarization through tight control of the cellular metabolic process (Byles et al., 2013; Covarrubias et al., 2015; Horng, 2015; Weichhart et al., 2015).

Internalization of microbes into phagosomes is a critical component of host defense. Once a macrophage internalizes the bacterium, then it can activate specific inflammasome formation, a response to cytosolic "danger signals" from the pathogens (Russell et al., 2009; Koizumi et al., 2012). The NOD-like receptor (NLR) family pyrin domain containing 3 (NLRP3) is critical for inflammasome activation. Activated caspase-1 cleaves pro-interleukin (IL)- $1 \beta$ to mature form IL- $1 \beta$ in response to microbial molecules, pyroptosis, and endogenous dangerassociated molecules (Schroder et al., 2010; Anand et al., 2011; Shi et al., 2017). We previously reported that $V$. vulnificus could directly induce macrophages to produce a variety of proinflammatory cytokines, such as tumor necrosis factor (TNF)- $\alpha$, IL-6, and IL-1 $\beta$ (Xie et al., 2017). Recent studies have shown that both NLRP3 inflammasome signaling and mTOR signaling in macrophages can be activated in response to bacterial cytotoxins upon V. vulnificus infection (Toma et al., 2010; Xie et al., 2017). Likewise, NLRP3 inflammasomes can be activated in response to other microbes, such as Salmonella typhimurium, Listeria monocytogenes, and Staphylococcus aureus (MunozPlanillo et al., 2009; Kim et al., 2010; Melehani et al., 2015; Pereira et al., 2016; Qu et al., 2016; O’Neill, 2017). Even though the relationship between innate immune signaling and phagocytosis in macrophages is well-established, the molecular mechanisms of bactericidal activity by macrophages against $V$. vulnificus infection remain poorly understood.

In this report, we found that the $V$. vulnificus infection boosted NLRP3 expression and mTORC1 activation. Endogenous NLRP3 interacted with mTOR in the macrophage. Knocking out NLRP3 impaired mTORC1 signaling, interfered phagolysosome formation by suppressing phagosome acidification, and resulted in insufficient intracellular V. vulnificus elimination. Moreover, inhibited mTORC1 signaling suppressed $V$. vulnificusinduced NLRP3 expression and caused insufficient phagosome acidification. Besides, mTORC1 signaling overactivation could increase NLRP3 expression and restore insufficient phagosome acidification. Our study demonstrates that intracellular bactericidal activity of macrophages against $V$. vulnificus infection is tightly controlled by the novel crosstalk of NLRP3 and mTOR, and it could be a new therapeutic approach for bacterial infections. Additionally, these findings provide evidence that NLRP3 signaling and mTORC1 signaling are integrated by protein-protein interaction between the signal-transducing intracellular elements from both pathways.

\section{RESULTS}

\section{Enhanced NLRP3 Expression by V. vulnificus Is Required for Intracellular Bacteria Elimination}

To understand how V. vulnificus causes inflammation and cell damage in macrophages, we performed RNA-Seq and transcriptomic analysis in J774A.1 cells before and after V. vulnificus infection. Compared with the infectious diseases gene pattern in the KEGG database, some genes responding to $V$. vulnificus infection were similar to those of 11 infectious diseases $(P<0.05)$, such as tuberculosis, salmonella infection, legionellosis, leishmaniasis, human cytomegalovirus (HCMV) infection, and influenza A infection (Figure 1A). Interestingly, as shown in Figure 1A, only one gene (IL-1 $\beta$ ) that responded to $V$. vulnificus infection was overlapping among tuberculosis, salmonella infection, legionellosis, leishmaniasis, HCMV infection, measles, and influenza A. Furthermore, only six genes ( $N l r p 3, C d 36, C c l 3$, etc.) overlapped in the core of the gene sets related to defense response, host response to the bacterium, innate immune response, IL- $1 \beta$ production, and regulation of the cellular metabolic process (Figure 1A). Next, we examined the expression of NLRP3 mRNA and protein in macrophages after $V$. vulnificus infection in vitro and in vivo. We found that NLRP3 mRNA expression was increased in Kupffer cells (KCs) from $V$. vulnificus-infected mice. Both NLRP3 mRNA expression and protein expression were elevated in bone marrow-derived macrophages $(B M M \phi s)$ and J774A.1 cells after $V$. vulnificus infection in vitro (Figures 1B,C). These data suggested that the expression of NLRP3 was enhanced after $V$. vulnificus infection.

To further investigate the essential role of NLRP3 in V. vulnificus-infected macrophages, we generated an NLRP3 
A
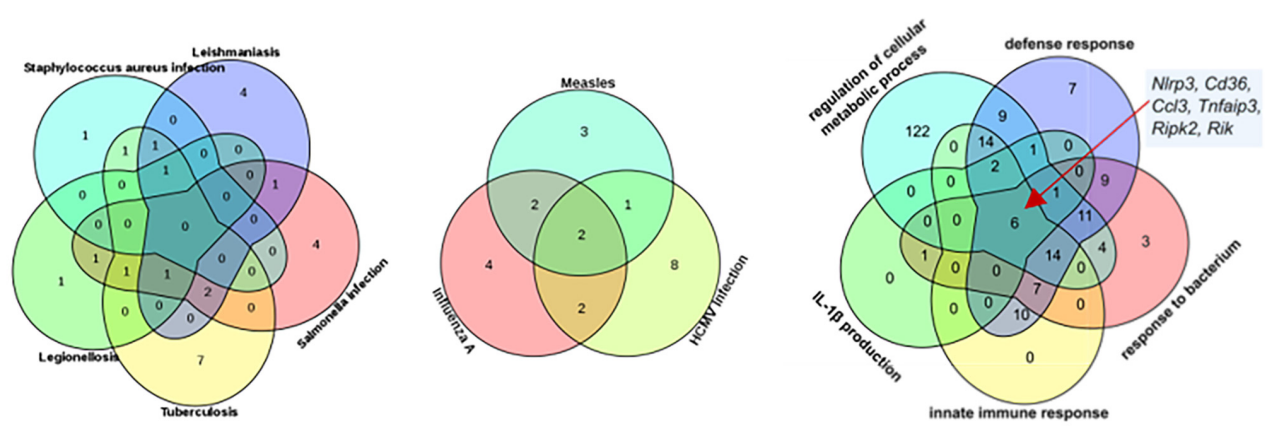

B

NIrp3

NIrp3
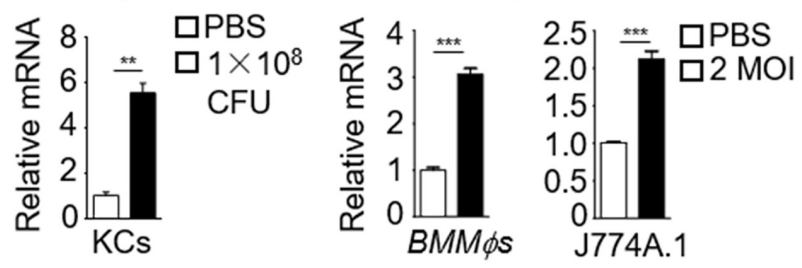

C

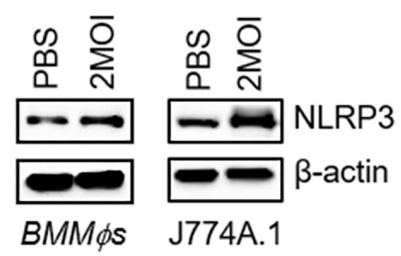

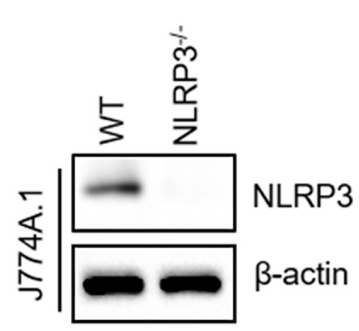

E

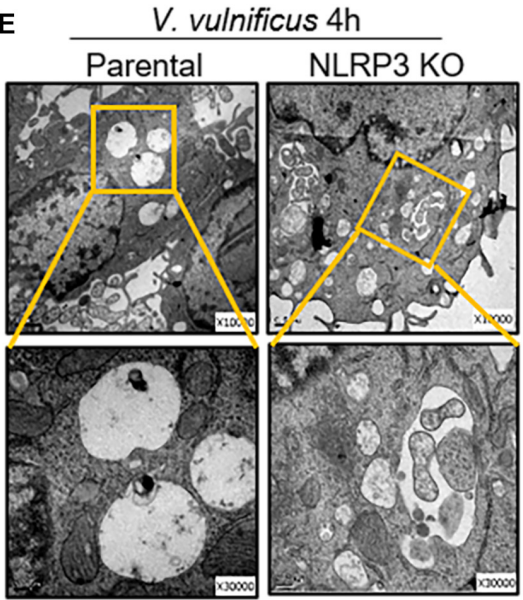

$\mathbf{F}$

Intracellular $V$. vulnificus titers

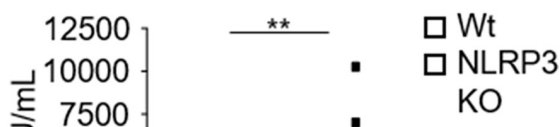

FIGURE 1 | The absence of NLRP3 impairs bactericidal activity in V. vulnificus-infected macrophages. (A) RNA-Seq analysis in PBS-treated or 2 MOI V. vulnificus-infected J774A.1 cells for $4 \mathrm{~h}$. The Venn diagrams shown are genes expressed in indicated infectious diseases (KEGG) and pathways (GO term) responding to $V$. vulnificus infection. The Venn diagram shows the overlap between each set of genes that are differentially expressed genes across indicated infectious diseases or host defense pathways $(P<0.05)$. (B) mRNA expression of NIrp3 in sorted KCs from $1 \times 10^{8}$ CFU V. vulnificus-infected mice or in 2 MOI V. vulnificus-infected BMM $\phi$ s and J774A. 1 cells. (C) NLRP3 protein expression in 2 MOI V. vulnificus-infected BMM $\phi s$ and J774A.1 cells. (D) Generation of an NLRP3-deficient J774A.1 cell line by CRISPR-Cas9 gene-editing system. Identification of the NLRP3 expression in biallelic deletion clones by Western blots. (E) Representative images of transmission electron microscopy are shown in the internalization of 2 MOI V. vulnificus in NLRP3-deficient and parental J774A. 1 cells for 4 h infection (top: original magnification $\times 10,000$, bottom: original magnification $\times 30,000$ ). (F) Intracellular microbial killing assay with viable $V$. vulnificus in parental and NLRP3 knockout J774A.1 cells after infection with $2 \mathrm{MOI}$ V. vulnificus for $4 \mathrm{~h}$. The cells were treated with $100 \mathrm{\mu g} / \mathrm{ml}$ gentamycin for 30 min to kill the attached bacteria. Then, the cells were lysed, and live bacteria in cell lysates were counted after incubation on BHI blood plates. Data (not including RNA-Seq data) shown are representative of at least three experiments. ${ }^{\star \star} P<0.01 ;{ }^{\star \star \star} P<0.001$ as determined by Student's $t$-test. RNASeq data are representative of at least two experiments.

knockout J774A.1 cell line using the CRISPR/Cas9 system. The J774A. 1 cells were transfected with a plasmid expressing a single guide RNA (sgRNA) targeting the endogenous mouse Nlrp3 exon two locus. The mutation caused a cleavage in the Nlrp3 locus at exon 2. Successful targeting was detected after PCR amplification of a 500 bp amplicon, flanking the target region of Nlrp3, 
reannealing of the PCR product, and selectively digesting the mismatched heteroduplex fragments by T7EI. T7EI recognizes and cleaves DNA mismatches in heteroduplexes. Homozygous NLRP3 KO or wild-type DNA alone shows the full-length band (about $500 \mathrm{bp}$ ), whereas heterozygous editing of NLRP3 alone or homozygous NLRP3 KO mixed with wild-type DNA shows a cleaved band. As shown in Supplementary Figure S1A, only NLRP3 KO mixed wild-type DNA showed a cleaved band, indicating that the mutant NLRP3 J774A.1 cells (KO cell) were homozygous. This mutation was further confirmed by DNA sequencing, which revealed a 10 bp deletion in exon 2, resulting in a frameshift mutation (Supplementary Figure S1B) and complete loss of NLRP3 expression (Figure 1D).

NLRP3-deficient J774A.1 cells contained more $V$. vulnificus than parental J774A.1 cells as observed in electron microscopy analysis (Figure 1E). The majority of internalized $V$. vulnificus in parental J774A.1 cells was degraded in the phagosome vacuole (Figure 1E). By contrast, intact V. vulnificus was observed inside phagosomes in the NLRP3-deficient J774A.1 cells (Figure 1E). Furthermore, more viable $V$. vulnificus could be detected in NLRP3-deficient J774A.1 cells than in parental cells (Figure 1F). This result indicates that NLRP3 restricts the intracellular growth of $V$. vulnificus in the macrophages. However, these results still cannot rule out whether $V$. vulnificus-caused parental cell damage is sufficiently disrupted to allow penetration by antibiotic to kill intracellular $V$. vulnificus. Next, we determined the cell viability of parental and NLRP3-deficient J774A.1 cells before and after $V$. vulnificus infection by flow cytometry. As shown in Supplementary Figures S2A,B, there is no significant difference between phosphate-buffered saline (PBS) and V. vulnificusinfected parental and NLRP3-deficient J774A.1 cells. Together, these results suggest that the NLRP3 regulation in the killing of intracellular bacteria in the macrophage is not due to cell death.

\section{Impaired Phagosome Acidification and Phagolysosomal Fusion Cause Compromised Degradation of Internalized $V$. vulnificus in NLRP3-Deficient Macrophages}

The elimination of bacteria by macrophages is a dynamic process involving bacteria internalization, phagosome formation, and phagosome and lysosome fusion (Kinchen and Ravichandran, 2008; Kim et al., 2019). The sufficient clearance of extracellular bacteria is dependent on both initial phagocytosis and functional phagolysosome (Buchmeier and Heffron, 1991; Wong et al., 2017). An acidic environment and the activation of $\mathrm{pH}$-sensitive enzymes contribute to degrading the internalized bacteria in the bactericidal function of macrophages (Kinchen and Ravichandran, 2008; Sedlyarov et al., 2018). Our transcriptomic analysis in J774A.1 cells revealed that the phagosome acidification pathway was significantly upregulated after $V$. vulnificus infection (Figure 2A). To investigate whether impaired intracellular bacterial killing capacity upon NLRP3 loss was correlated with phagosome acidification damage. Lysosenor Green DND 189 staining (which measures the $\mathrm{pH}$ value of acidic organelles) was used to test phagosome acidification. We found that phagosomes containing $V$. vulnificus did not acidify sufficiently in NLRP3deficient J774A.1 cells (Figure 2B). The phagosomes acidified to $\mathrm{pH}<4$ after $4 \mathrm{~h}$ post-infection in parental J774A.1 cells (Figure 2C), whereas the phagosomes acidified to $\mathrm{pH}>4$ in V. vulnificus-infected NLRP3 KO cell (Figure 2C). These results suggested that NLRP3 was required for maintaining sufficient phagosome acidification to clear the intracellular V. vulnificus. We also tested the mRNA expression of phagosome acidificationrelated genes, including Rap1a, Rab20, Rab27a, and Atp6v1h. The mRNA expression of these genes was significantly decreased in V. vulnificus-infected NLRP3-deficient J744A.1 cells (Figure 2D).

A new phagosome undergoes a process termed "maturation" by fusion and limited fission events with endosomes and lysosomes to generate an acidic and highly hydrolytic mature phagolysosome (Kinchen and Ravichandran, 2008). Phagocytosis and phagosome maturation eventually lead to progressive acidification of the phagolysosome. The low $\mathrm{pH}$ value in the mature phagolysosome is required for the ingestion and elimination of invading pathogens. Then, we determined whether losing NLRP3 caused a failure of phagolysosome formation. Endocytic trafficking to lysosomes in J774A.1 cells was examined by incubation of macrophages with Alexa Fluor 546 dextran for $2 \mathrm{~h}$. The internalization of pHrodo Escherichia coli BioParticles by J774A.1 cells strongly suggested phagosomes. Phagolysosome formation, including the maturation of phagosomes fused with lysosomes, was determined by an analysis of the colocalization of dextran and pHrodo E. coli BioParticles. As shown in Figures 2E,F, in the parental J774A.1 cells, dextran staining was partially colocalizing with internalized pHrodo E. coli BioParticles, indicating the fusion of lysosome and phagosome. However, there is almost no colocalization of dextran with pHrodo E. coli BioParticles in NLRP3-deficient J774A.1 cells, which meant a blockage in phagolysosome formation. Together, our results suggested a disruption of phagosome acidification and Gram-negative bacteria-mediated phagolysosome formation with NLRP3 deficiency in macrophages.

\section{mTORC1 and NLRP3 Reciprocally Regulate V. vulnificus-Infected Macrophage Bactericidal Activity}

mTOR is a master kinase that regulates lysosome structure and function in lysosomal biogenesis, distribution, and activity (Puertollano, 2014). mTOR also localizes to the lysosomal membrane, which is a crucial regulator for the cellular metabolism homeostasis (Wang et al., 2017; Rabanal-Ruiz and Korolchuk, 2018). To address this issue, we analyzed mTORC1 signaling activity in NLRP3-deficient and parental J774A.1 cells with various stimulation. Incubated with $V$. vulnificus, E. coli, lipopolysaccharide (LPS), or serum, the NLRP3-deficient J774A.1 cells showed comparable increased phosphorylation levels in both mTOR and S6K1 (Figure 3A). Moreover, lysates of rapamycin-treated macrophages were analyzed for mTORC1 signaling. The protein phosphorylation level of mTOR, S6K, S6, and 4E-BP1 in V. vulnificus-infected J774A.1 cells treated with rapamycin was dramatically decreased compared with those cells without rapamycin (Figure 3B). Besides, we observed an 
A

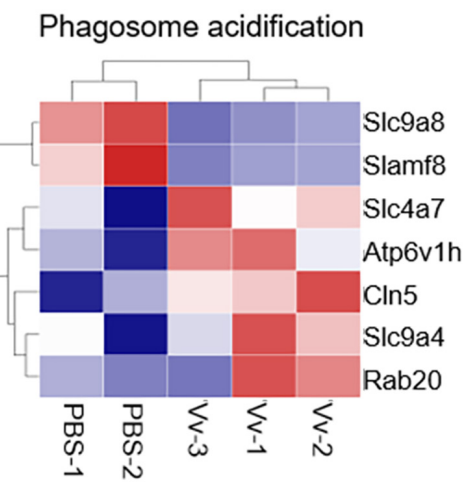

C

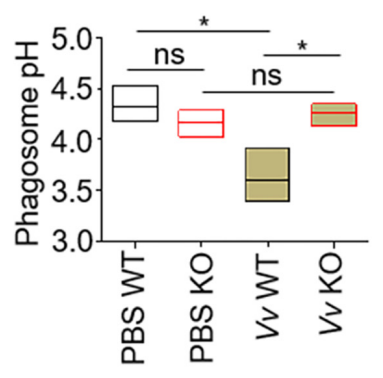

B

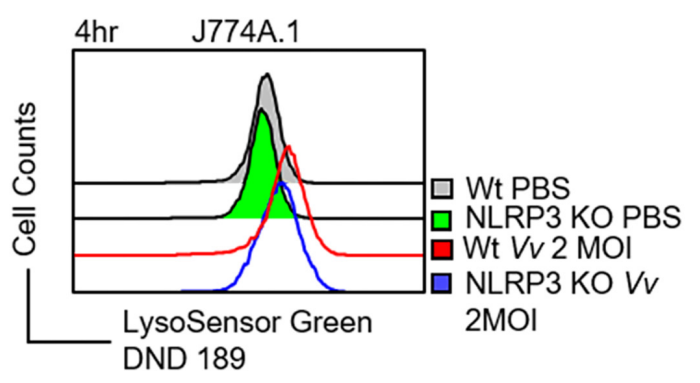

D
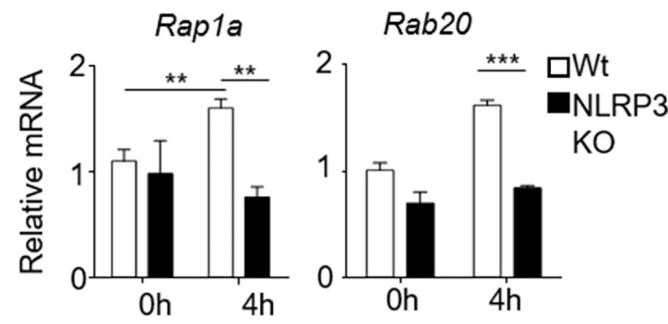

Rab27a

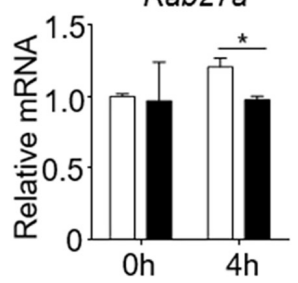

Atp6v1h

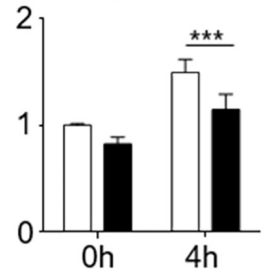

E

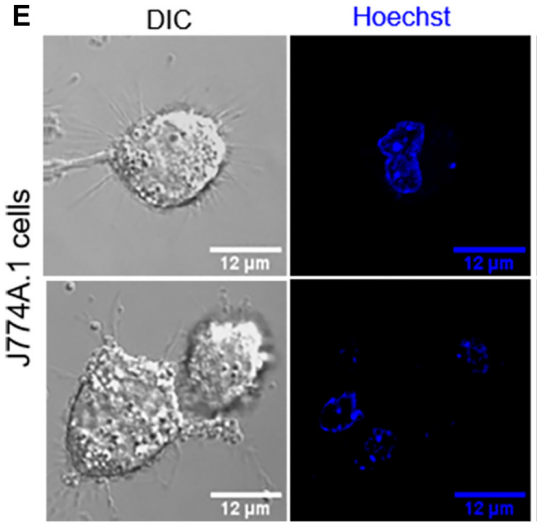

Alexa Fluor 546- pHrodo Red E. coli

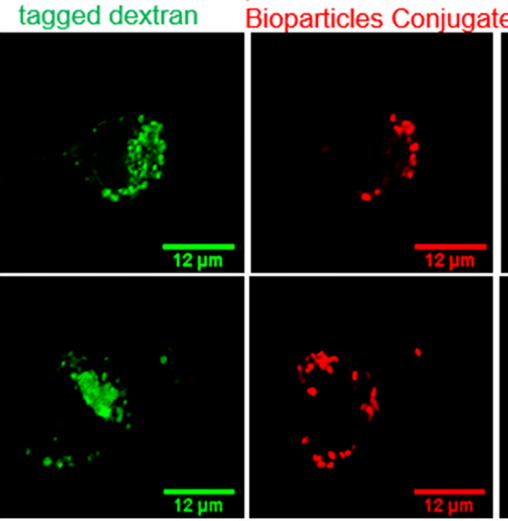

Merge

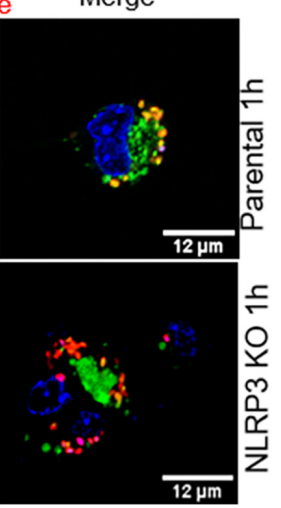

F

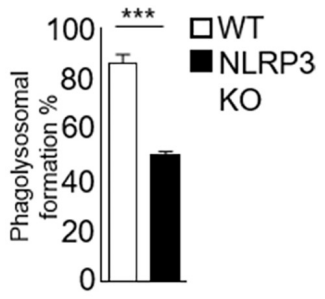

FIGURE 2 | NLRP3 is crucial for regulation of phagosome acidification and phagolysosome formation in $V$. vulnificus-infected macrophages. (A) Heatmap of significantly enriched gene set in phagosome acidification from V. vulnificus-infected and uninfected J774A.1 cells $(P<0.05)$. (B) Phagosome acidification was detected by $\mathrm{pH}$ indicator dye LysoSensor Green DND-189 staining. After 4 h of infection with 2 MOI V. vulnificus, the cells were stained with $1 \mu \mathrm{M}$ LysoSensor Green DND-189 and then subjected to flow cytometry analysis. (C) The phagosome pH in parental and NLRP3-deficient J774A.1 cells was measured by LysoSensor Yellow/Blue DND-160. Phagosome $\mathrm{pH}$ values were determined from calibration curves generated from permeabilized cells in various pH buffers. (D) RT-PCR analysis of mRNA expression of genes in phagosome maturation and acidification. (E) Confocal images are shown in phagolysosome formation. The Alexa Fluor 546 conjugated dextran was ingested by macrophage through endocytosis. The cells were cultured for $4 \mathrm{~h}$ to let the endosome develop to a mature lysosome. The pHrodo Red E. coil BioParticles conjugate uptake by the cells through phagocytosis. The phagolysosome formation is visualized by phagosome and lysosome fusion. Scale bar: $12 \mu \mathrm{M}$. (F) The bar figure shown is the percentage of the phagosome and lysosome fusion in NLRP3-deficient and parental J774A.1 cells after 2 MOI V. vulnificus infection. Data (not including RNA-Seq data) shown are representative of at least three experiments. ${ }^{\star} P<0.05$; ${ }^{\star \star} P<0.01$; ${ }^{\star \star \star} P<0.001$ as determined by Student's $t$-test. RNASeq data are representative of at least two experiments. 
A

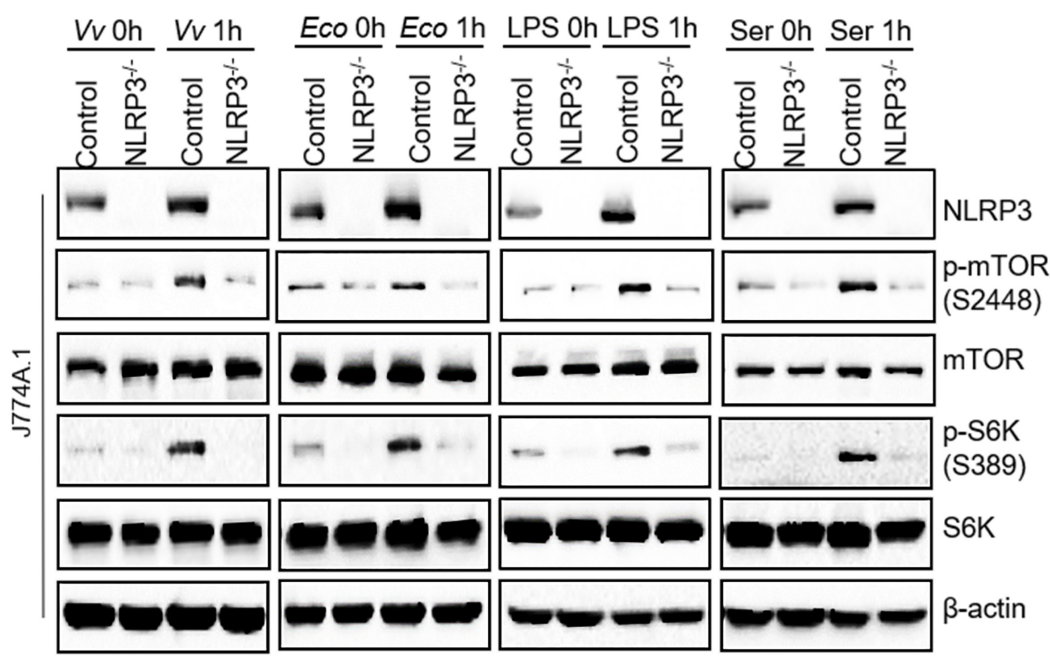

B

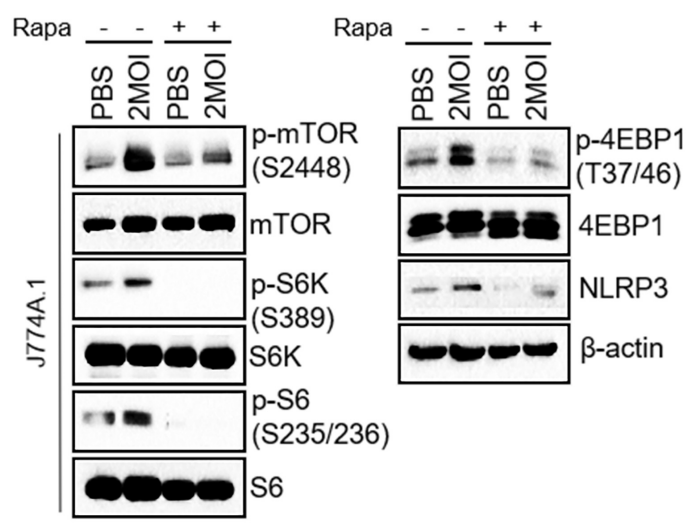

C

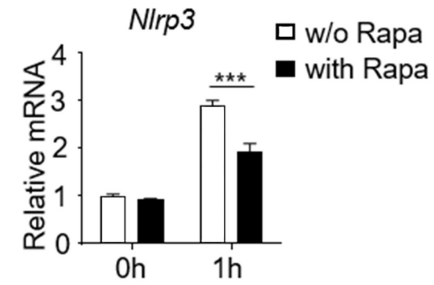

D $\square$ w/o Rapamycin $\square$ with Rapamycin

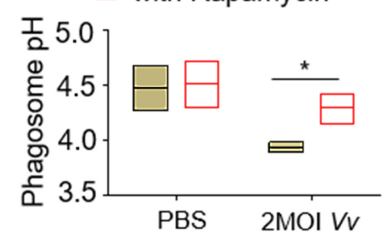

E

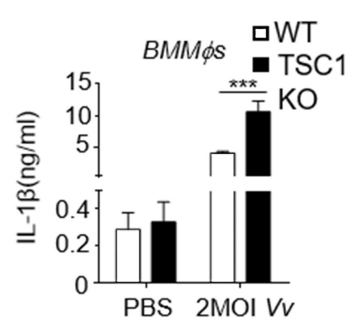

F w/o Rapa with Rapa

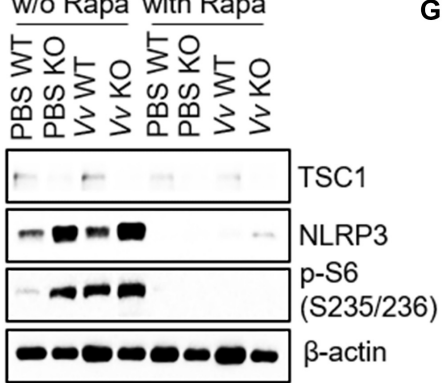

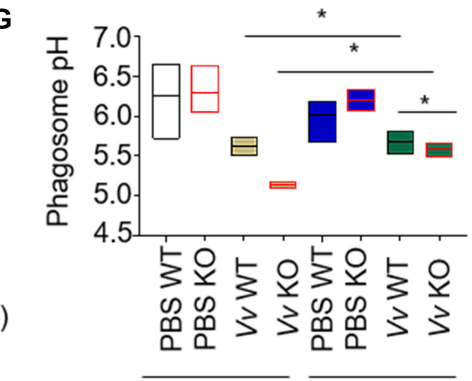

$\begin{array}{cc}\text { w/o } & \text { With } \\ \text { Rapamycin } & \text { Rapamycin }\end{array}$

FIGURE 3 | Reciprocal regulation of NLRP3 and mTORC1 impairs phagosome acidification in V. vulnificus-infected macrophages. (A) Western blot analysis of mTOR signaling in parental and NLRP3 knockout J774A.1 cells upon different stimulations. (B) Western blot analysis of mTOR signaling and NLRP3 expression in J774A.1 cells with indicated treatments. (C) RT-PCR analysis of NIrp3 mRNA expression in the J774A.1 cells with or without rapamycin after V. vulnificus infection. (D) $\mathrm{pH}$ values of phagosomes in the indicated J774A.1 cells. (E) IL-1 $\beta$ secretion in the supernatant of WT and TSC1 KO BMM $\phi S$ after $V$. vulnificus infection for $4 \mathrm{~h}$. (F) Western blot analysis of mTOR signaling and NLRP3 expression in $2 \mathrm{MOI} V$. vulnificus-infected or -uninfected wild-type and TSC1 KO BMM $\$ S$ with or without 100 nM rapamycin. (G) Phagosome pH in WT and TSC1 KO BMM $\phi s$ was measured by LysoSensor Yellow/Blue DND-160. Data shown are representative of at least three experiments. Rapa, rapamycin; WT, C57BL/6J wild-type mice; TSC1 KO, TSC1 ${ }^{\text {flox } / \text { flox }}$ LysM-Cre ${ }^{+}$mice. ${ }^{*} P<0.05 ;{ }^{* \star *} P<0.001$ as determined by Student's t-test. 
A

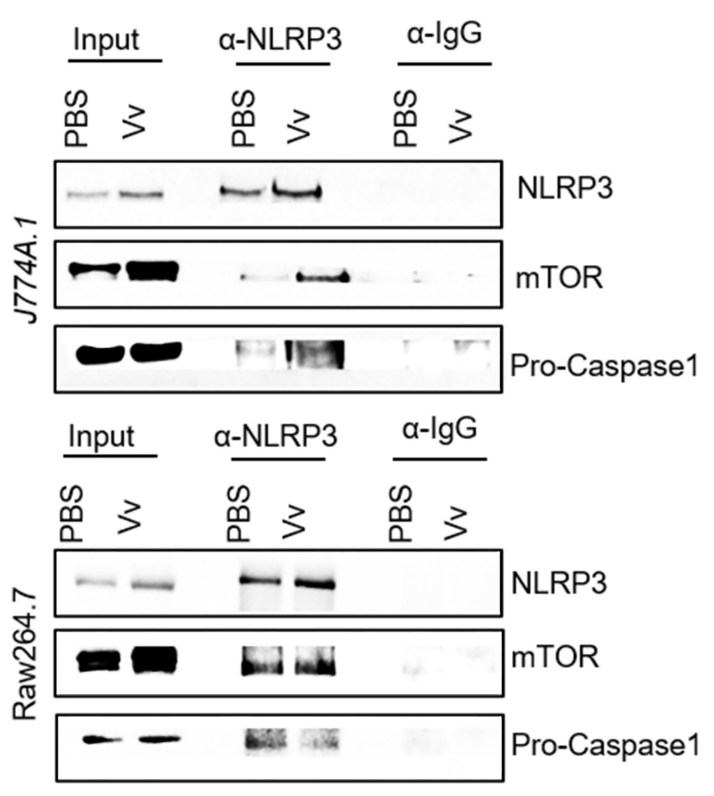

B
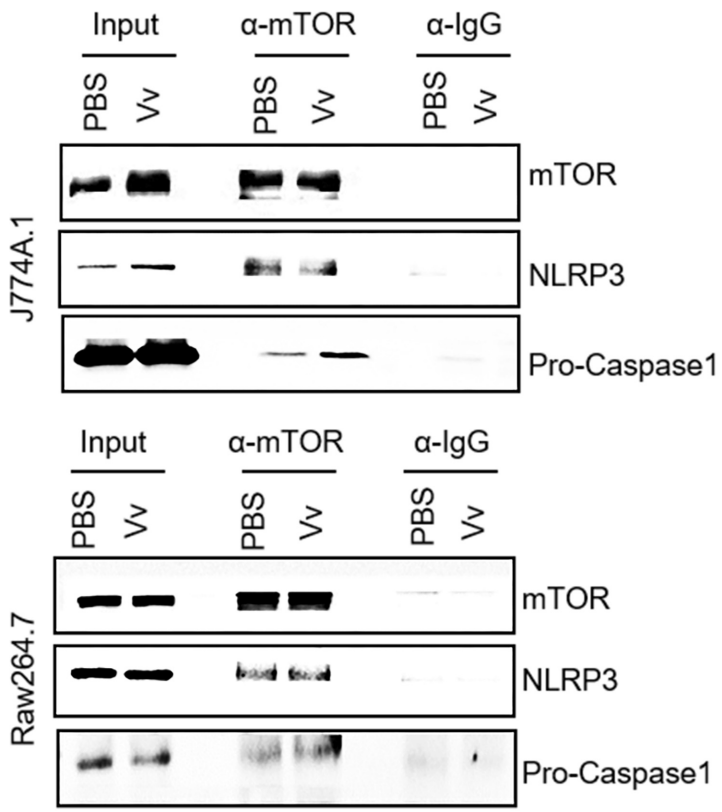
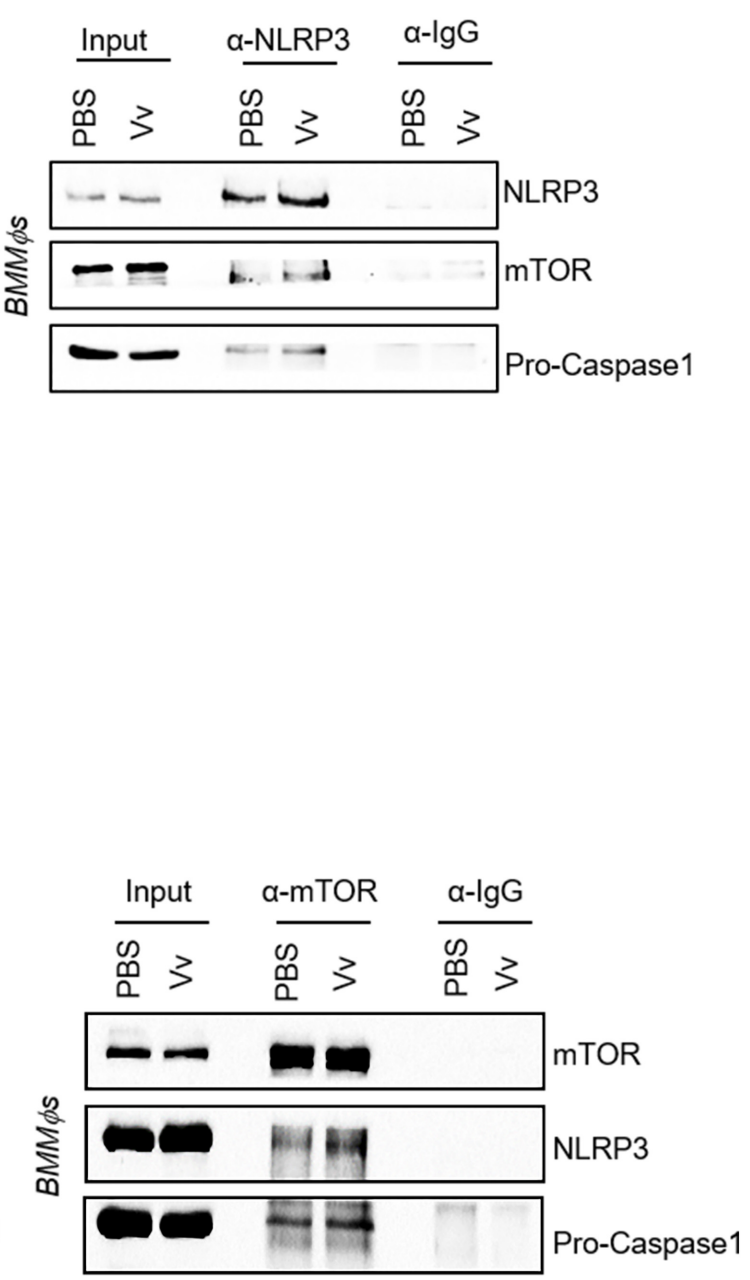

FIGURE 4 | Interaction between NLRP3 and mTOR in murine macrophages. (A,B) Whole-cell lysates from PBS-treated or V. vulnificus-infected J774A.1 cells, Raw264.7 cells, and BMM $\phi$ s were immunoprecipitated with NLRP3 antibody (A) or mTOR antibody (B), and Western blots were performed with antibodies against NLRP3, mTOR, and pro-caspase-1. Data shown are representative of at least three experiments.

additional inhibitory effect on the expression of NLRP3 in both mRNA and protein levels in J774A.1 cells after $V$. vulnificus infection (Figures 3B,C). To avoid the NLRP3 KO clone could result from just clone to clone variation rather than loss of NLRP3. We also detected other clones of NLRP3 KO J774A.1 cells (Supplementary Figure S3A). The mTOR signaling was also impaired in NLRP3 KO cells (Supplementary Figure S3B). These data demonstrated that $V$. vulnificus-induced mTORC1 activation and NLRP3 expression could be inhibited by rapamycin. Besides, we observed a significantly elevated $\mathrm{pH}$ value in rapamycin-treated parental J774A.1 cells (Figure 3D).

To further address the relationship between mTORC1 overactivation and NLRP3 expression during $V$. vulnificus infection, we infected the $B M M \phi s$ from Tuberous Sclerosis Complex 1 (TSC1) flox/flox $^{\text {LysM-Cre }}{ }^{+}$mice $(\mathrm{TSC} 1 \mathrm{KO}$ ) and C57BL/6J (wild-type, WT) mice with 2 multiplicity of infection (MOI) V. vulnificus. Infected TSC1 KO BMM $\phi$ s increased the production of mature IL- $1 \beta$ in response to $V$. vulnificus challenge 
(Figure 3E). As shown in Figure 3E, S6 phosphorylation increased in TSC1 KO BMM 1 , indicating that mTORC1 signaling overactivated. S6 phosphorylation in both wild-type and TSC1 KO BMM 1 s was increased after $V$. vulnificus infection (Figure 3F). However, NLRP3 expression increased in $V$. vulnificus-uninfected TSC1 KO BMM $\phi s$ and V. vulnificusinfected WT $B M M \phi s$, indicating that mTORC1 overactivation could enhance NLRP3 expression, which could be reversed by rapamycin treatment (Figure $3 \mathbf{F}$ ). Besides, the $\mathrm{pH}$ value in V. vulnificus-infected TSC1-deficient $B M M \phi s$ was much lower than that in WT BMM $\phi$ s (Figure 3G). However, by comparing with J774A.1 cells (Figure 3D), the $\mathrm{pH} B M M \phi s$ was much higher, suggesting that the $\mathrm{pH}$ value could be variable in different cell lines. These results indicated that there was crosstalk between NLRP3 and mTORC1 in phagosome acidification.

\section{Endogenous NLRP3 Interacts With mTOR in Macrophages}

To investigate the underlying mechanism of NLRP3 and mTOR reciprocal regulation of phagosome acidification and host bactericidal activity in macrophages during $V$. vulnificus infection, we conducted an immunoprecipitation assay in V. vulnificus-infected or -uninfected WT BMM $\phi$ s, J774A.1 cells, and Raw264.7 cells using anti-NLRP3 or anti-mTOR monoclonal antibody. We found that endogenous NLRP3 interacted with mTOR not only in J774A.1 cells but also in Raw264.7 cells and WT BMM $\phi$ s (Figure 4A). The $V$. vulnificus infection could enhance the interaction between NLRP3 and mTOR by the upregulation of NLRP3 expression, indicating that the proteinprotein interaction was likely affected by $V$. vulnificus infection (Figure 4A). In the total cell lysate (input), the NLRP3 band slightly shifted after immunoprecipitation (Figure 4A). The band from SDS-PAGE lanes was used to confirm whether it was NLRP3 by mass spectrometry (liquid chromatography with tandem mass spectrometry, LC-MS/MS). The LC-MS/MS results confirmed that the hit with the highest score of peptides was NLRP3 (Supplementary Figure S4 and Supplementary Table S2). Moreover, we used an anti-mTOR monoclonal antibody to pull down endogenous mTOR. The blots showed that mTOR interacted with NLRP3 in J774A.1 cells, Raw264.7 cells, and WT BMM申s (Figure 4B). Additionally, we observed that the interaction between NLRP3 and NLRP3 inflammasome components (e.g., pro-caspase-1) or mTOR and pro-caspase1 simultaneously existed (Figures 4A,B), which indicated that pro-caspase-1 might be constitutively associated with NLRP3. Collectively, these data highlight mTOR as a novel component of the NLRP3 inflammasome, and the reciprocal regulation between mTORC1 and NLRP3 is likely via protein-protein interaction. However, the role of mTOR in association with NLRP3 is still not currently understood.

\section{DISCUSSION}

Although much of the recent work has linked the pathogenicity of $V$. vulnificus infection to innate and adaptive immune response, inflammasome activation, cytotoxicity of virulence factors (e.g., cell death and inflammation), and host iron metabolism, little is known about how host phagocytes eliminate pathogens encountering V. vulnificus (Jones and Oliver, 2009; Toma et al., 2010; Pajuelo et al., 2014; Arezes et al., 2015; Lee et al., 2017; Xie et al., 2017; Baker-Austin and Oliver, 2018). Our study made several advances in illuminating the crosstalk between NLRP3 and mTOR in the regulation of bactericidal activity, including phagosome acidification, phagolysosome formation, and intracellular bacterial clearance in macrophage during V. vulnificus infection. Our results suggested a novel mechanism about the reciprocal regulation of NLRP3 and mTORC1 signaling in phagolysosome-mediated intracellular microbial killing in V. vulnificus-infected macrophages.

NLRP3 is involved in acidification of phagosomes bearing Gram-positive but not Gram-negative organisms (Sokolovska et al., 2013). However, we found that Gram-negative bacteria, such as $V$. vulnificus, also boost NLRP3 expression. The absence of NLRP3 could cause insufficient intracellular bacterial clearance via impaired lysosomal function, phagosome acidification, and phagolysosome formation. Our results bolster a recent study (Sokolovska et al., 2013) that links caspase-1 to regulate phagosome acidification and phagolysosome formation. By deletion of NLRP3, the phagosome failed to rapidly acidify. The insufficient phagosome acidification allows the sustained survival of $V$. vulnificus in the phagocytes and results in the delay of invoked microbial degradation. Here, we show that NLRP3 deficiency in macrophages impairs the expression of various genes linked to phagosome acidification and maturation, including Rap1a, Rab27a, Rab20, and Atp6v1h. Previous studies have shown that a GTPase family protein, Rap1A, was localized to late endosome/phagosome compartments in J774 macrophages by colocalization with LAMP2, which may be involved in late endocytic/phagocytic processes (Chung et al., 2008). Rab27a also belongs to the family of small GTPase, which is localized on dendritic cell-phagosomes and is implicated in the regulation of phagosome acidification in dendritic cells (Jancic et al., 2007). Rab20 is enriched on the phagosome, which is likely essential for phagosome maturation. Phagosome maturation includes the acquisition of vacuolar ATPase, a proton pump involved in the maintenance of intracellular organellar acidification (Kinchen and Ravichandran, 2008; Russell et al., 2009). A mature form of phagosome is crucial for phagolysosomal fusion, and phagolysosomes display sufficient antimicrobial capacity. Thus, the V-type proton ATPase complex, such as Atp6ap1, Atp6v1a, and Atp6v1h, is thought to be a dynamic marker for phagolysosomal fusion (Xiong and Zhu, 2016). Together, our results extend the previous findings from Sokolovska et al. (2013). Our study shows that NLRP3 is likely essential for operating the phagosome and lysosome fusion processes through small GTPase and V-type proton ATPase complex genes.

Moreover, our results show that rapamycin completely blocks the phosphorylation of S6 in $V$. vulnificus-infected macrophages. The $\mathrm{pH}$ in $V$. vulnificus-infected TSC1 KO macrophages is still lower than that in WT macrophages in the presence of rapamycin. It suggests that TSC1 likely regulates $\mathrm{pH}$ in $V$. vulnificus-infected macrophages in an mTOR-independent manner. Whether this mTOR-independent manner is involved in 
regulating phagosome acidification still needs to be determined in the future. Recent studies have also revealed that mTORC1 activity is important for phagosomes to undergo late maturation (Krajcovic et al., 2013). Additionally, the activation of NLRP3 inflammasome leads to the leakage of lysosomal contents into the cytosol after phagocytosis of particulates (Amaral et al., 2018). Alternatively, the release of reactive oxygen species (ROS) into the cytosol during lysosomal membrane permeabilization also could trigger NLRP3 inflammasome activation (Heid et al., 2013). Thus, the mechanisms underlying NLRP3 inflammasome, mTOR activity, and phagosome/lysosome in defense of Gram-negative bacterial infection still require clarification. Our results found a protein-protein interaction between NLRP3 and mTOR in macrophages, and the absence of NLRP3 significantly inhibits mTORC1 activation. Whereas, increasing mTORC1 activation by deletion of TSC1 in macrophages resulted in enhanced NLRP3 expression, which could be reversed by rapamycin. Besides, inhibited mTORC1 activation by rapamycin also reduced the NLRP3 protein expression, following increased phagosome $\mathrm{pH}$. Our results indicated that mTOR kinase activity was likely involved in NLRP3 stability/post-translation through protein-protein interaction. Cosin-Roger et al. (2017) also reported NLRP3 as a novel binding partner of mTOR in HT-29 cells regulated by hypoxia. In our results in correspondence with their results, we found that the physical interaction of NLRP3 and mTOR exists in both macrophage cell lines (J774A.1 cells and RAW264.7 cells) and primary macrophages $(B M M \phi s)$. This interaction plays an essential role against $V$. vulnificus infection. Whereas NLRP3 lacks a caspase recruitment domain (CARD), it cannot recruit pro-caspase1 except in the presence of the adaptor molecule apoptosisassociated speck-like protein containing a CARD (ASC) (Davis et al., 2011). Interestingly, RAW264.7 cells do not have the ASC adapter, indicating that pro-caspase-1 would have to directly associate with NLRP3 or another complex component (e.g., mTOR), not through the ASC adapter. Future studies will be needed to clarify whether the interaction of NLRP3 and $\mathrm{mTOR}$ in macrophages plays a crucial role in pathogen clearance in vivo.

Li et al. (2018) have found that mTORC1/2 inhibitor INK128 decreases both mRNA and protein levels of NLRP3 in LPS/ATP and LPS/nigericin-stimulated murine BMM $\phi s$. Additionally, Nakahira's group has reported that Torin 1 suppresses mTOR1/2 activity, which inhibits NLRP3 inflammasome activation through hexokinase-1 (HK-1) glycolysis in macrophages (Moon et al., 2015). These previous studies indicate that mTOR kinase activity is likely involved in NLRP3 mRNA transcription and protein expression. We found that the inhibition of mTORC1 activation by rapamycin could suppress $V$. vulnificus-induced NLRP3 expression in both mRNA and protein levels. Our results not only are consistent with these previous studies but also strengthen the role of mTORC1 in the regulation of NLRP3 expression. Moreover, our results provide new clues for the relationship between mTOR and NLRP3 through protein-protein interaction in the macrophages. Whether mTOR-mediated HK1-dependent glycolysis is involved in the regulation of $V$. vulnificus-induced NLRP3 expression is still needed to be determined in the future.
Overall, our results indicate that the reciprocal regulation of mTOR and NLRP3 is important for intracellular $V$. vulnificus clearance in macrophages. Activated mTOR signaling and upregulated NLRP3 allow macrophages to limit internalized microbes in the host cells and establish efficient phagolysosomal function in defense of $V$. vulnificus infection. Whether the NLRP3-mTOR reciprocal regulation plays a similar role in the modulation of host defense to other Gram-negative bacteria or intracellular microbe infection remains to be addressed.

\section{MATERIALS AND METHODS}

\section{Mice}

Six- to eight-week-old C57BL/6J mice were purchased from the Chinese Academy of Sciences, Shanghai Laboratory Animal Center (SLAC). Tsc1 flox mice and Lysozyme Cre transgenic mice were obtained from the Jackson Laboratory. Tsc1 flox mice were bred with Lysozyme Cre mice to induce TSC1 deletion in myeloid cells. All the mice were maintained and used in accordance with the animal experimental guidelines set by the Wenzhou Medical University Animal Care and Use Committee.

\section{Bacterial Strains and Cell Culture}

L-929 cells and J774A.1 cells were purchased from the Cell Bank of Type Culture Collection of Chinese Academy of Sciences in Shanghai. All the cells were cultured in RPMI 1640 containing $10 \%$ heat-inactivated fetal bovine serum (FBS, Ausvin) and penicillin-streptomycin (50 IU/ml and $50 \mathrm{mg} / \mathrm{ml}$, Beyotime). The China General Microbiological Culture Collection Center provided the $V$. vulnificus CGMCC 1.1758 strain. We grew V. vulnificus at $37^{\circ} \mathrm{C}$ in brain heart infusion (BHI) broth or on the BHI rabbit blood agar plate. The procedures of $V$. vulnificus that were used in this study were followed from the standard biological hazards at Biosafety Level 2 and the safety procedures of Wenzhou Medical University Laboratory Safety Department.

\section{Generation of Bone Marrow-Derived Macrophages}

The bone marrow cells were isolated from femurs and tibiae and cultured with a 10\% L-929 cell culture medium, as described previously (Pan et al., 2012). After 3 days of culturing at $37^{\circ} \mathrm{C}$ in a $\mathrm{CO}_{2}$ incubator, non-adherent cells were collected and transferred into new Petri dishes with a freshly conditioned medium. After another 4 days of culture, the BMM $\phi s$ were ready for experimentation. Flow cytometry was used to analyze the $\mathrm{F} 4 / 80^{+} \mathrm{CD} 1 \mathrm{~b}^{+}$cell purity. Over $90 \%$ of the cells were $\mathrm{F} 4 / 80^{+} \mathrm{CD} 11 \mathrm{~b}^{+}$cells.

\section{Bacterial Treatment of $B M M \phi s$ and J774A.1 Cells in vitro}

$B M M \phi s$ and J774A.1 cells were plated in $35 \mathrm{~mm}$ dishes, and then $V$. vulnificus was added to the cells at the indicated MOI. The supernatant was collected at the indicated time for cytokine quantification. The infected cells were used for flow cytometry analysis, confocal microscopy analysis, 
quantitative reverse transcription PCR (RT-qPCR) analysis, and Western blot analysis.

\section{Phagosome Acidification}

To measure phagosome acidification, the cells were treated with $1 \mu \mathrm{M}$ LysoSensor Green DND-189 for $30 \mathrm{~min}$ at $37^{\circ} \mathrm{C}$ and then were analyzed by flow cytometry. Additionally, to obtain a $\mathrm{pH}$ standard curve, the cells were loaded with LysoSensor Yellow/Blue DND-160 for $5 \mathrm{~min}$ at $37^{\circ} \mathrm{C}$; the lysosomal $\mathrm{pH}$ was equalized by incubating cells for $5 \mathrm{~min}$ at $37^{\circ} \mathrm{C}$ before incubating in a calibration buffer $(125 \mathrm{mM}$ $\mathrm{KCl}, 25 \mathrm{mM} \mathrm{NaCl}, 10 \mu \mathrm{M}$ monensin, and $25 \mathrm{mM} \mathrm{4-}$ morpholineethanesulfonic acid hydrate (MES) with the $\mathrm{pH}$ adjusted to either $3.5,4.0,4.5,5.0,5.5,6.0$, or 6.5 , respectively). Then, the cells were incubated at the same time points that in generating the $\mathrm{pH}$ calibration curve and washed twice with PBS. Data were collected with a microplate reader by excitation at 440 and $540 \mathrm{~nm}$.

\section{Phagolysosome Formation}

The phagolysosome formation was determined as previously described (Baker et al., 2015). Briefly, the cells were incubated with Alexa Fluor 488-tagged dextran $(250 \mu \mathrm{g} / \mathrm{ml})$ for $1 \mathrm{~h}$ after $1 \mu \mathrm{g} / \mathrm{ml}$ Hoechst 33342 staining (Beyotime). The cells were then washed three times and incubated in RPMI 1640 medium for $3 \mathrm{~h}$ to allow lysosome translocation of dextran. The cells were subjected to incubation with pHrodo Red E. coli BioParticles for $1 \mathrm{~h}$. Data were collected using a Nikon A1 confocal microscope.

\section{Transcriptome Profiling}

J774A.1 cells with $4 \mathrm{~h} \quad V$. vulnificus infection or PBS treatment were used for transcriptome sequencing by Annoroad Co. Significantly differentially expressed genes were identified when we compared the normalized reads count between $V$. vulnificus and PBS groups with $P<0.05$ and $\mid \log 2$ FoldChange $>0.263$. The significance of the gene ontology term enrichment was estimated using Fisher's Exact Test $(P$-value).

\section{Real-Time qPCR Analysis}

Total RNA was extracted from sorted KCs, BMMфs, and J774A.1 cells by TRIzol reagent (Omega Bio-Tek). cDNA was synthesized using the FastQuant RT Kit (TIANGEN). Real-time qPCR was performed as previously described (Xie et al., 2012). Target mRNA expression was normalized with $\beta$-actin and then was calculated using the $2^{-\Delta \Delta C T}$ method. Primers were shown in Supplementary Table S1.

\section{Western Blot Analysis}

Cell lysates preparation and Western blot analysis were performed by following the previous protocol (Xie et al., 2012). Antibodies against p-mTOR, mTOR, NLRP3, p-S6K, p-4EBP1, p-S6, $\beta$-actin, S6K, S6, and 4EBP1 were purchased from Cell Signaling Technology (CST).

\section{Generation of an NLRP3-Deficient J774A.1 Cell Line}

Murine Nlrp3 guide RNA was designed using an online tool ${ }^{1}$. By following the previously described protocol (Ran et al., 2013), the annealed oligo (Forward, 5'-CAC CGA CAT TCC TCT ATG GTA TGC C-3'; Reverse, 5'-AAA CGG CAT ACC ATA GAG GAA TGT C-3') was cloned into pSpCas9 (BB)-2A-GFP (PX458) vector (Addgene) to generate PX458-NLRP3 plasmid. Then, PX458-NLRP3 plasmid was transfected into J774A.1 cells using the QuickShuttle-Superfast Transfection Kit (Biodragon Immunotech) following the manufacturer's protocol.

\section{Flow Cytometry}

Fluorochrome-conjugated anti-CD11b and anti-F4/80 were purchased from Biolegend. Cell death was identified using a violet Live/Dead Kit or 7AAD staining (Invitrogen). The cells were stained with fluorescence conjugated antibodies in $2 \%$ (vol/vol) $\mathrm{FBS}$ in PBS at $4^{\circ} \mathrm{C}$ for $30 \mathrm{~min}$. Data were collected using a BD FACSAria II and analyzed using the FlowJo software. CD11b ${ }^{\text {int }} \mathrm{F} 4 / 80^{+}$KCs from C57BL/6J mice were sorted using a BD FACSAria II sorter.

\section{Electron Microscopy}

Electron microscopic analysis was performed as previously described (Lee et al., 2017). Briefly, the cells were washed with PBS and harvested into a $1.5 \mathrm{ml}$ Eppendorf tube. The cells were then fixed with $2.5 \%$ glutaraldehyde, post-fixed in $1 \%$ osmium tetroxide and 1\% potassium, and embedded in Quetol 812. Ultrathin sections were stained with uranyl acetate and lead nitrate. Images were observed and acquired using an H-7500 Hitachi transmission electron microscope.

\section{Immunoprecipitation}

J774A.1 cells, RAW264.7 cells, and $B M M \phi s$ were infected with 2 MOI $V$. vulnificus. The cells were lysed for total protein using Pierce ${ }^{\mathrm{TM}}$ IP Lysis Buffer (Thermo Fisher Scientific) containing protease and phosphatase inhibitors (Sigma). In total, $500 \mu \mathrm{g}$ cell lysate was incubated with $6 \mu \mathrm{l}$ anti-mTOR antibody (CST) or anti-NLRP3 antibody (CST) or $2 \mu \mathrm{l}$ antirabbit IgG (CST) overnight at $4^{\circ} \mathrm{C}$ to form the complex. The immunoprecipitation assay was performed using the Magnetic IP Kit (Thermo Fisher Scientific) according to the manufacturer's protocol. Briefly, the antigen-antibody mixture was incubated with prewashed magnetic beads for $1 \mathrm{~h}$ and washed with wash buffer twice. Then, the beads were collected using a magnetic stand, eluted in a low-pH elution buffer, and then used for Western blot analysis.

\section{Intracellular Bacterial Growth}

The cells were infected with 2 MOI $V$. vulnificus for $4 \mathrm{~h}$ and washed with PBS. The supernatant was diluted and plated on blood BHI plates for $12 \mathrm{~h}$. Then, $100 \mu \mathrm{g} / \mathrm{ml}$ gentamicin was added to kill extracellular bacteria. After $30 \mathrm{~min}$, the cells were washed twice, treated with $0.1 \%$ Triton X-100, and plated on blood BHI plates for $12 \mathrm{~h}$ at $37^{\circ} \mathrm{C}$ for a bacterial count.

\footnotetext{
${ }^{1}$ http://crispr.mit.edu
} 


\section{Statistical Analysis}

Data were presented as mean \pm SEM and analyzed for statistical differences using the Prism 6.01/GraphPad software. Statistical significance was analyzed using the Student's $t$-test. $P$-values less than 0.05 were considered significant.

\section{DATA AVAILABILITY STATEMENT}

The raw data supporting the conclusions of this article will be made available by the authors, without undue reservation, to any qualified researcher.

\section{ETHICS STATEMENT}

The animal study was reviewed and approved by the Wenzhou Medical University Animal Care and Use Committee.

\section{AUTHOR CONTRIBUTIONS}

D-LX, Y-LL, and YZ conceived and designed the experiments. D-LX, Y-LL, YZ, X-HH, YM, M-MZ, NC, M-NH, and L-YW analyzed the data. D-LX, YZ, X-HH, YM, M-MZ, NC, M-NH, and L-YW conducted the experiments. D-LX and X-HH wrote the manuscript. All authors contributed to the article and approved the submitted version.

\section{REFERENCES}

Amaral, E. P., Riteau, N., Moayeri, M., Maier, N., Mayer-Barber, K. D., Pereira, R. M., et al. (2018). Lysosomal cathepsin release is required for NLRP3-inflammasome activation by Mycobacterium tuberculosis in infected macrophages. Front. Immunol. 9:1427. doi: 10.3389/fimmu.2018.01427

Anand, P. K., Malireddi, R. K., and Kanneganti, T. D. (2011). Role of the nlrp3 inflammasome in microbial infection. Front. Microbiol. 2:12. doi: 10.3389/ fimmu.2018.00012

Arezes, J., Jung, G., Gabayan, V., Valore, E., Ruchala, P., Gulig, P. A., et al. (2015). Hepcidin-induced hypoferremia is a critical host defense mechanism against the siderophilic bacterium Vibrio vulnificus. Cell Host Microb. 17, 47-57. doi: 10.1016/j.chom.2014.12.001

Baker, B., Geng, S., Chen, K., Diao, N., Yuan, R., Xu, X., et al. (2015). Alteration of lysosome fusion and low-grade inflammation mediated by super-lowdose endotoxin. J. Biol. Chem. 290, 6670-6678. doi: 10.1074/jbc.m114.6 11442

Baker-Austin, C., and Oliver, J. D. (2018). Vibrio vulnificus: new insights into a deadly opportunistic pathogen. Environ. Microbiol. 20, 423-430. doi: 10.1111/ 1462-2920.13955

Baker-Austin, C., and Oliver, J. D. (2020). Vibrio vulnificus. Trends Microbiol. 28, 81-82.

Bauernfeind, F. G., Horvath, G., Stutz, A., Alnemri, E. S., MacDonald, K., Speert, D., et al. (2009). Cutting edge: NF-kappaB activating pattern recognition and cytokine receptors license NLRP3 inflammasome activation by regulating NLRP3 expression. J. Immunol. 183, 787-791. doi: 10.4049/jimmunol.09 01363

Benoit, M., Desnues, B., and Mege, J. L. (2008). Macrophage polarization in bacterial infections. J. Immunol. 181, 3733-3739. doi: 10.4049/jimmunol.181. 6.3733

Blake, P. A., Merson, M. H., Weaver, R. E., Hollis, D. G., and Heublein, P. C. (1979). Disease caused by a marine Vibrio. Clinical characteristics and epidemiology. N. Engl. J. Med. 300, 1-5. doi: 10.1056/nejm197901043000101

\section{FUNDING}

This work was supported by the National Natural Science Foundation of China (31400764 to D-LX, 81772229 to Y-LL), the Provincial Natural and Science Foundation of Zhejiang (LY13H190007 to D-LX), the Wenzhou Municipal Science and Technology Bureau (Y20150113 to D-LX), the Science Foundation of Zhejiang Provincial Education Department (Y201636809 to YZ), and the National Science and Technology Major Projects of China (2018ZX10201001-009 to Y-LL).

\section{ACKNOWLEDGMENTS}

We appreciate the Light Microscope Core Facility and Flow Cytometry Core Facility at the Wenzhou Medical University for the service and Wei-Tao Cong from Proteomics and Metabolomics Core Facility in the Wenzhou Medical University for protein identification service.

\section{SUPPLEMENTARY MATERIAL}

The Supplementary Material for this article can be found online at: https://www.frontiersin.org/articles/10.3389/fcell.2020. 587961/full\#supplementary-material

Buchmeier, N. A., and Heffron, F. (1991). Inhibition of macrophage phagosomelysosome fusion by Salmonella typhimurium. Infect. Immun. 59, 2232-2238. doi: 10.1128/iai.59.7.2232-2238.1991

Byles, V., Covarrubias, A. J., Ben-Sahra, I., Lamming, D. W., Sabatini, D. M., Manning, B. D., et al. (2013). The TSC-mTOR pathway regulates macrophage polarization. Nat. Commun. 4:2834.

Chou, T. N., Lee, Y. T., Lai, Y. Y., Chao, W. N., Yang, C., Chen, C. C., et al. (2010). Prognostic factors for primary septicemia and wound infection caused by Vibrio vulnificus. Am. J. Emerg. Med. 28, 424-431. doi: 10.1016/j.ajem.2008.12.037

Chung, J., Serezani, C. H., Huang, S. K., Stern, J. N., Keskin, D. B., Jagirdar, R., et al. (2008). Rap1 activation is required for Fc gamma receptor-dependent phagocytosis. J. Immunol. 181, 5501-5509. doi: 10.4049/jimmunol.181.8.5501

Cosin-Roger, J., Simmen, S., Melhem, H., Atrott, K., Frey-Wagner, I., Hausmann, M., et al. (2017). Hypoxia ameliorates intestinal inflammation through NLRP3/mTOR downregulation and autophagy activation. Nat. Commun. 8:98.

Covarrubias, A. J., Aksoylar, H. I., and Horng, T. (2015). Control of macrophage metabolism and activation by mTOR and Akt signaling. Semin. Immunol. 27, 286-296. doi: 10.1016/j.smim.2015.08.001

Davis, B. K., Wen, H., and Ting, J. P. (2011). The inflammasome NLRs in immunity, inflammation, and associated diseases. Annu. Rev. Immunol. 29, 707-735. doi: 10.1146/annurev-immunol-031210-101405

Deng, W., Yang, J., Lin, X., Shin, J., Gao, J., and Zhong, X. P. (2017). Essential role of mTORC1 in Self-renewal of murine alveolar macrophages. J. Immunol. 198, 492-504. doi: 10.4049/jimmunol.1501845

Diacovich, L., and Gorvel, J. P. (2010). Bacterial manipulation of innate immunity to promote infection. Nat. Rev. Microbiol. 8, 117-128. doi: 10.1038/ nrmicro2295

Gulig, P. A., Bourdage, K. L., and Starks, A. M. (2005). Molecular pathogenesis of Vibrio vulnificus. J. Microbiol. 43, 118-131.

Heid, M. E., Keyel, P. A., Kamga, C., Shiva, S., Watkins, S. C., and Salter, R. D. (2013). Mitochondrial reactive oxygen species induces NLRP3-dependent lysosomal damage and inflammasome activation. J. Immunol. 191, 5230-5238. doi: 10.4049/jimmunol.1301490 
Heng, S. P., Letchumanan, V., Deng, C. Y., Ab Mutalib, N. S., Khan, T. M., Chuah, L. H., et al. (2017). Vibrio vulnificus: an environmental and clinical burden. Front. Microbiol. 8:997. doi: 10.3389/fimmu.2018.00997

Horng, T. (2015). mTOR trains heightened macrophage responses. Trends Immunol. 36, 1-2. doi: 10.1016/j.it.2014.11.004

Ishii, K. J., Koyama, S., Nakagawa, A., Coban, C., and Akira, S. (2008). Host innate immune receptors and beyond: making sense of microbial infections. Cell Host Microb. 3, 352-363. doi: 10.1016/j.chom.2008.05.003

Jancic, C., Savina, A., Wasmeier, C., Tolmachova, T., El-Benna, J., Dang, P. M., et al. (2007). Rab27a regulates phagosomal $\mathrm{pH}$ and NADPH oxidase recruitment to dendritic cell phagosomes. Nat. Cell Biol. 9, 367-378. doi: 10.1038/ncb1552

Jeong, H. G., and Satchell, K. J. (2012). Additive function of Vibrio vulnificus MARTX(Vv) and VvhA cytolysins promotes rapid growth and epithelial tissue necrosis during intestinal infection. PLoS Pathog. 8:e1002581. doi: 10.1371/ journal.ppat.1002581

Jones, M. K., and Oliver, J. D. (2009). Vibrio vulnificus: disease and pathogenesis. Infect. Immun. 77, 1723-1733. doi: 10.1128/iai.01046-08

Kim, B. R., Kim, B. J., Kook, Y. H., and Kim, B. J. (2019). Phagosome escape of rough Mycobacterium abscessus strains in murine macrophage via phagosomal rupture can lead to Type I interferon production and their cell-to-cell spread. Fron.t Immunol. 10:125. doi: 10.3389/fimmu.2018.00125

Kim, S., Bauernfeind, F., Ablasser, A., Hartmann, G., Fitzgerald, K. A., Latz, E., et al. (2010). Listeria monocytogenes is sensed by the NLRP3 and AIM2 inflammasome. Eur. J. Immunol. 40, 1545-1551. doi: 10.1002/eji.201040425

Kinchen, J. M., and Ravichandran, K. S. (2008). Phagosome maturation: going through the acid test. Nat. Rev. Mol. Cell Biol. 9, 781-795. doi: 10.1038/nrm2515

Koizumi, Y., Toma, C., Higa, N., Nohara, T., Nakasone, N., and Suzuki, T. (2012). Inflammasome activation via intracellular NLRs triggered by bacterial infection. Cell Microbiol. 14, 149-154. doi: 10.1111/j.1462-5822.2011.01707.x

Krajcovic, M., Krishna, S., Akkari, L., Joyce, J. A., and Overholtzer, M. (2013). mTOR regulates phagosome and entotic vacuole fission. Mol. Biol. Cell. 24, 3736-3745. doi: 10.1091/mbc.e13-07-0408

Lee, S. J., Jung, Y. H., Kim, J. S., Lee, H. J., Lee, S. H., Lee, K. H., et al. (2017). A Vibrio vulnificus VvpM induces IL-1beta production coupled with necrotic macrophage death via distinct spatial targeting by ANXA2. Front. Cell Infect. Microbiol. 7:352. doi: 10.3389/fimmu.2018.00352

Li, X., Zhang, X., Pan, Y., Shi, G., Ren, J., Fan, H., et al. (2018). mTOR regulates NLRP3 inflammasome activation via reactive oxygen species in murine lupus. Acta Biochim. Biophys. Sin. 50, 888-896. doi: 10.1093/abbs/g my088

Melehani, J. H., James, D. B., DuMont, A. L., Torres, V. J., and Duncan, J. A. (2015). Staphylococcus aureus leukocidin A/B (LukAB) kills human monocytes via host NLRP3 and ASC when extracellular, but not intracellular. PLoS Pathog. 11:e1004970. doi: 10.1371/journal.ppat.1004970

Moon, J. S., Hisata, S., Park, M. A., DeNicola, G. M., Ryter, S. W., Nakahira, K., et al. (2015). mTORC1-induced HK1-dependent glycolysis regulates NLRP3 inflammasome activation. Cell Rep. 7, 102-115. doi: 10.1016/j.celrep.2015. 05.046

Munoz-Planillo, R., Franchi, L., Miller, L. S., and Nunez, G. (2009). A critical role for hemolysins and bacterial lipoproteins in Staphylococcus aureus-induced activation of the Nlrp3 inflammasome. J. Immunol. 183, 3942-3948. doi: 10. 4049/jimmunol.0900729

O’Neill, L. A. J. (2017). The hunger games: Salmonella, anorexia, and NLRP3. Cell Metab. 25, 225-226. doi: 10.1016/j.cmet.2017.01.015

Pajuelo, D., Lee, C. T., Roig, F. J., Lemos, M. L., Hor, L. I., and Amaro, C. (2014). Host-nonspecific iron acquisition systems and virulence in the zoonotic serovar of Vibrio vulnificus. Infect. Immun. 82, 731-744. doi: 10.1128/iai.01117-13

Pan, H., O'Brien, T. F., Zhang, P., and Zhong, X. P. (2012). The role of tuberous sclerosis complex 1 in regulating innate immunity. J. Immunol. 188, 3658-3666. doi: 10.4049/jimmunol.1102187

Park, J., and Lee, C. S. (2018). Vibrio vulnificus infection. N. Engl. J. Med. 379:375.

Pereira, M., Tourlomousis, P., Wright, J., Pm, T., and Bryant, C. E. (2016). CARD9 negatively regulates NLRP3-induced IL-1beta production on Salmonella infection of macrophages. Nat. Commun. 7:12874.

Puertollano, R. (2014). mTOR and lysosome regulation. F1000Prime Rep. 6:52.
Qu, Y., Misaghi, S., Newton, K., Maltzman, A., Izrael-Tomasevic, A., Arnott, D., et al. (2016). NLRP3 recruitment by NLRC4 during Salmonella infection. J. Exp. Med. 213, 877-885. doi: 10.1084/jem.20132234

Rabanal-Ruiz, Y., and Korolchuk, V. I. (2018). mTORC1 and nutrient homeostasis: the central role of the Lysosome. Int. J. Mol. Sci. 19:818. doi: 10.3390/ ijms19030818

Ran, F. A., Hsu, P. D., Wright, J., Agarwala, V., Scott, D. A., and Zhang, F. (2013). Genome engineering using the CRISPR-Cas9 system. Nat. Protoc. 8, 2281-2308.

Russell, D. G., Vanderven, B. C., Glennie, S., Mwandumba, H., and Heyderman, R. S. (2009). The macrophage marches on its phagosome: dynamic assays of phagosome function. Nat. Rev. Immunol. 9, 594-600. doi: 10.1038/nr i2591

Schroder, K., Zhou, R., and Tschopp, J. (2010). The NLRP3 inflammasome: a sensor for metabolic danger? Science 327, 296-300. doi: 10.1126/science.11 84003

Sedlyarov, V., Eichner, R., Girardi, E., Essletzbichler, P., Goldmann, U., NunesHasler, P., et al. (2018). The bicarbonate transporter SLC4A7 plays a key role in macrophage phagosome acidification. Cell Host Microb. 23, 766-774. doi: 10.1016/j.chom.2018.04.013

Shi, J., Gao, W., and Shao, F. (2017). Pyroptosis: gasdermin-mediated programmed necrotic cell death. Trends Biochem. Sci. 42, 245-254. doi: 10.1016/j.tibs.2016. 10.004

Sokolovska, A., Becker, C. E., Ip, W. K., Rathinam, V. A., Brudner, M., Paquette, N., et al. (2013). Activation of caspase-1 by the NLRP3 inflammasome regulates the NADPH oxidase NOX2 to control phagosome function. Nat. Immunol. 14, 543-553. doi: 10.1038/ni.2595

Toma, C., Higa, N., Koizumi, Y., Nakasone, N., Ogura, Y., McCoy, A. J., et al. (2010). Pathogenic Vibrio activate NLRP3 inflammasome via cytotoxins and TLR/nucleotide-binding oligomerization domain-mediated NF-kappa B signaling. J. Immunol. 184, 5287-5297. doi: 10.4049/jimmunol.09 03536

Wang, A., Carraro-Lacroix, L. R., Owen, C., Gao, B., Corey, P. N., Tyrrell, P., et al. (2017). Activity-independent targeting of mTOR to lysosomes in primary osteoclasts. Sci. Rep. 7:3005.

Weichhart, T., Hengstschlager, M., and Linke, M. (2015). Regulation of innate immune cell function by mTOR. Nat. Rev. Immunol. 15, 599-614. doi: 10.1038/ nri3901

Wong, C. O., Gregory, S., Hu, H., Chao, Y., Sepulveda, V. E., He, Y., et al. (2017). Lysosomal degradation is required for sustained phagocytosis of bacteria by macrophages. Cell Host Microb. 21, 719-730. doi: 10.1016/j.chom.2017.05.002

Xie, D. L., Wu, J., Lou, Y. L., and Zhong, X. P. (2012). Tumor suppressor TSC1 is critical for T-cell anergy. Proc. Natl. Acad. Sci. U.S.A. 109, 14152-14157. doi: 10.1073/pnas.1119744109

Xie, D. L., Zheng, M. M., Zheng, Y., Gao, H., Zhang, J., Zhang, T., et al. (2017). Vibrio vulnificus induces mTOR activation and inflammatory responses in macrophages. PLoS One 12:e0181454. doi: 10.1371/journal.ppat.0171454

Xiong, J., and Zhu, M. X. (2016). Regulation of lysosomal ion homeostasis by channels and transporters. Sci. China Life Sci. 59, 777-791. doi: 10.1007/ s11427-016-5090-x

Zhao, H., Xu, L., Dong, H., Hu, J., Gao, H., Yang, M., et al. (2015). Correlations between clinical features and mortality in patients with Vibrio vulnificus infection. PLoS One 10:e0136019. doi: 10.1371/journal.ppat.0136019

Conflict of Interest: The authors declare that the research was conducted in the absence of any commercial or financial relationships that could be construed as a potential conflict of interest.

Copyright (C) 2020 Huang, Ma, Zheng, Chen, Hu, Wu, Zheng, Lou and Xie. This is an open-access article distributed under the terms of the Creative Commons Attribution License (CC BY). The use, distribution or reproduction in other forums is permitted, provided the original author(s) and the copyright owner(s) are credited and that the original publication in this journal is cited, in accordance with accepted academic practice. No use, distribution or reproduction is permitted which does not comply with these terms. 